
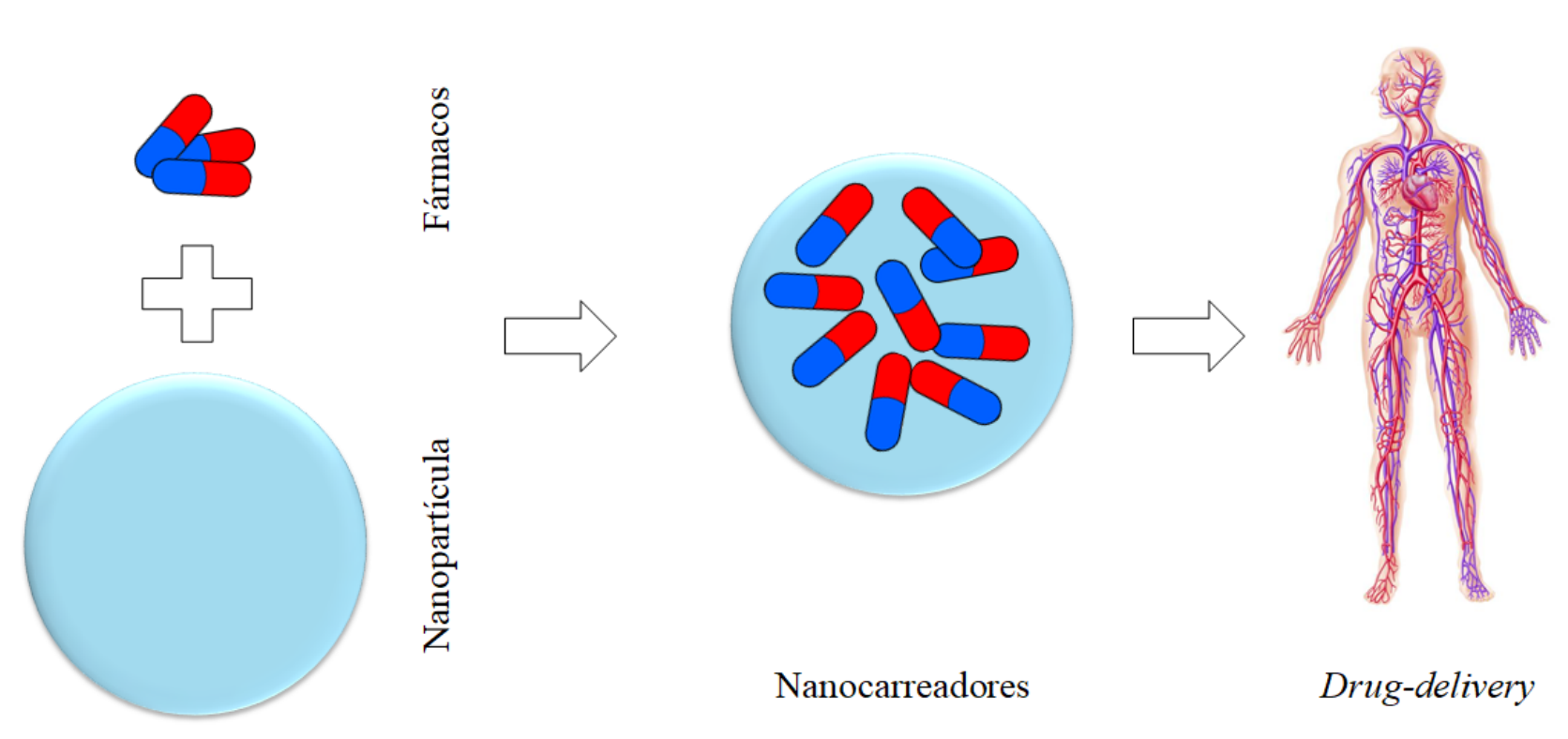

\title{
SISTEMAS DE ENTREGA DE FÁRMACOS: FUNDAMENTOS E AVANÇOS
}

\author{
Jéssica T. dos Santos*, Beatriz F. dos Santos, Marcel L. de Almeida, Koiti Araki* \\ Instituto de Química, Universidade de São Paulo (USP), São Paulo, SP
}

*e-mail: jessica.teixeira@usp.br, koiaraki@iq.usp.br 


\section{SISTEMAS DE ENTREGA DE FÁRMACOS: FUNDAMENTOS E AVANÇOS}

Resumo: Os sistemas de entrega de fármacos, ou nanocarreadores, são materiais biocompatíveis que, em conjunto com os compostos bioativos adequados, atuam como um sistema de entrega de drogas. Esses materiais têm sido amplamente estudados nos últimos anos, principalmente devido a biodegradabilidade, não toxicidade e facilidade de ajustar suas características para uma determinada finalidade. Assim, neste artigo de revisão é dada uma visão geral dos fundamentos da nanotecnologia com foco nos diferentes tipos de nanocarreadores, apresentando algumas definições e conceitos, bem como as características que possibilitam seu uso na entrega de fármacos para as células-alvo. Além disso, os desafios e as perspectivas futuras para os sistemas de entrega de medicamentos também são discutidos.

Palavras-Chave: nanotecnologia, nanocarreadores, nanopartículas, nanocápsulas, lipossomas.

\section{DRUG DELIVERY SYSTEMS: CONCEPTS AND ADVANCES}

Abstract: Drug delivery systems, or nanocarriers, are biocompatible materials that loaded with suitable bioactive compounds act as drug delivery systems. These materials have been widely studied in recent years, mainly due to characteristics such as biodegradability, nontoxicity and ease to adjust key features aiming a particular purpose. Thus, an overview on the fundamentals of nanotechnology focusing on the main types of nanocarriers is provided in this review article, presenting some definitions and concepts, and the characteristics that enable their use in the delivery of drugs to target cells as well. In addition, the challenges and future perspectives for drug delivery systems are also discussed.

Keywords: nanotechnology, nanocarriers, nanoparticles, nanocapsules, lipossomes. 


\section{INTRODUÇÃO}

O prefixo grego "nano" significa simplesmente "anão" e representa um bilionésimo do metro $\left(10^{-9} \mathrm{~m}\right)$, dimensões muito pequenas, completamente fora do alcance de nossa visão, ou de microscópios ópticos, mas que estão sendo ativamente exploradas utilizando-se microscópios que utilizam feixes de elétrons (ao invés de luz visível) para gerar as imagens. Assim, nanomateriais são definidos como materiais que apresentam pelo menos uma de suas dimensões na escala de cerca de 1 a $100 \mathrm{~nm}$, que podem ser produzidos subdividindose materiais convencionais (estratégia top-down), ou construindo-se a partir de átomos ou moléculas (estratégia bottom-up). ${ }^{1}$ Considerando-se um cubo de $1 \mathrm{~cm}$ de lado, percebe-se claramente que se cortarmos o mesmo gerando folhas de $1 \mathrm{~nm}$ de espessura obteremos 10.000.000 (dez milhões) de folhas com mais ou menos a área de duas de suas faces. Se estes forem cortadas novamente na espessura de $1 \mathrm{~nm}$, cada folha gerará dez milhões de bastões de $1 \mathrm{~cm}^{2}$ e $1 \mathrm{~cm}$ de comprimento (Figura 1). Finalmente, se repetirmos o procedimento com esses bastões cada um deles irá produzir dez milhões de nanocubos de 1 $\mathrm{nm}^{3}$ cada um. Note, que neste processo a área superficial está sendo aumentada enormemente à medida que subdividimos o cubo gerando nanomateriais, ou seja, nanofolhas, nanobastões e nanocubos. ${ }^{2}$ Outro aspecto muito importante que deve ser lembrado é que no processo de corte do material, estamos quebrando ligações e gastando energia. Logo, estamos gerando superfícies de alta energia e altamente reativas que tendem a se juntar novamente, ou seja, de agregar gerando partículas maiores. Note que as nanopartículas como as que descrevemos não são entidades estáveis e precisam ser funcionalizadas, ou seja, moléculas devem ser ligadas a superfície não somente diminuindo a energia de superfície, mas atribuindo-lhes novas propriedades de interação. ${ }^{3,4}$ 


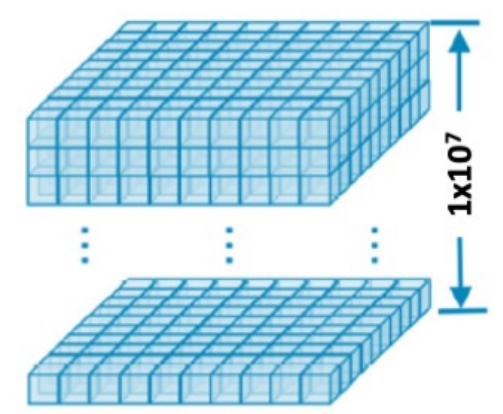

Cubo

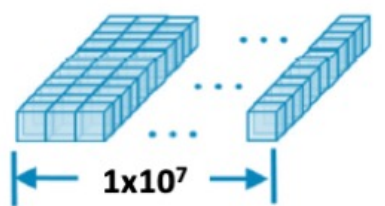

Folha

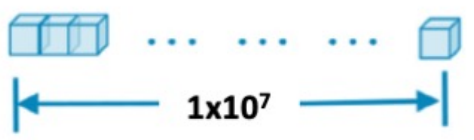

Fio

Figura 1. Esquemas ilustrando o aumento da área superficial a medida que um cubo de 1 $\mathrm{cm}$ de lado é cortado em $1 \times 10^{7}$ folhas de $1 \mathrm{~nm}$ de espessura e estes em $1 \times 10^{14}$ bastões de 1 $\mathrm{nm}^{2}$ de base, que finalmente podem ser cortados para gerar $1 \times 10^{21} \mathrm{cubos}$ de $1 \mathrm{~nm}^{3}$.

A nanotecnologia é considerada uma das últimas, se não a última fronteira do conhecimento, e visa o desenvolvimento de novos materiais e aplicações a partir da manipulação, montagem, controle de materiais com pelo menos uma de suas dimensões na escala nanométrica (Figura 2). $\mathrm{O}$ desenvolvimento de processos de fabricação suficientemente simples, economicamente competitivos e ambientalmente amigáveis de nanomateriais possibilitará sua aplicação na resolução de problemas relevantes da sociedade e agregação de valor nos mais diversos segmentos, como de energia, saúde e meio ambiente, de maneira sustentável, além de estudos inovadores nas áreas de química, física, engenharia, e biologia, dentre outras. Mas, para isso é fundamental o domínio do processo de preparação, incluindo o domínio da química de superfície/funcionalização, para que possamos controlar as propriedades das nanopartículas, por exemplo, ajustando-as para o desenvolvimento de aplicações na área biomédica. ${ }^{5,6}$ 


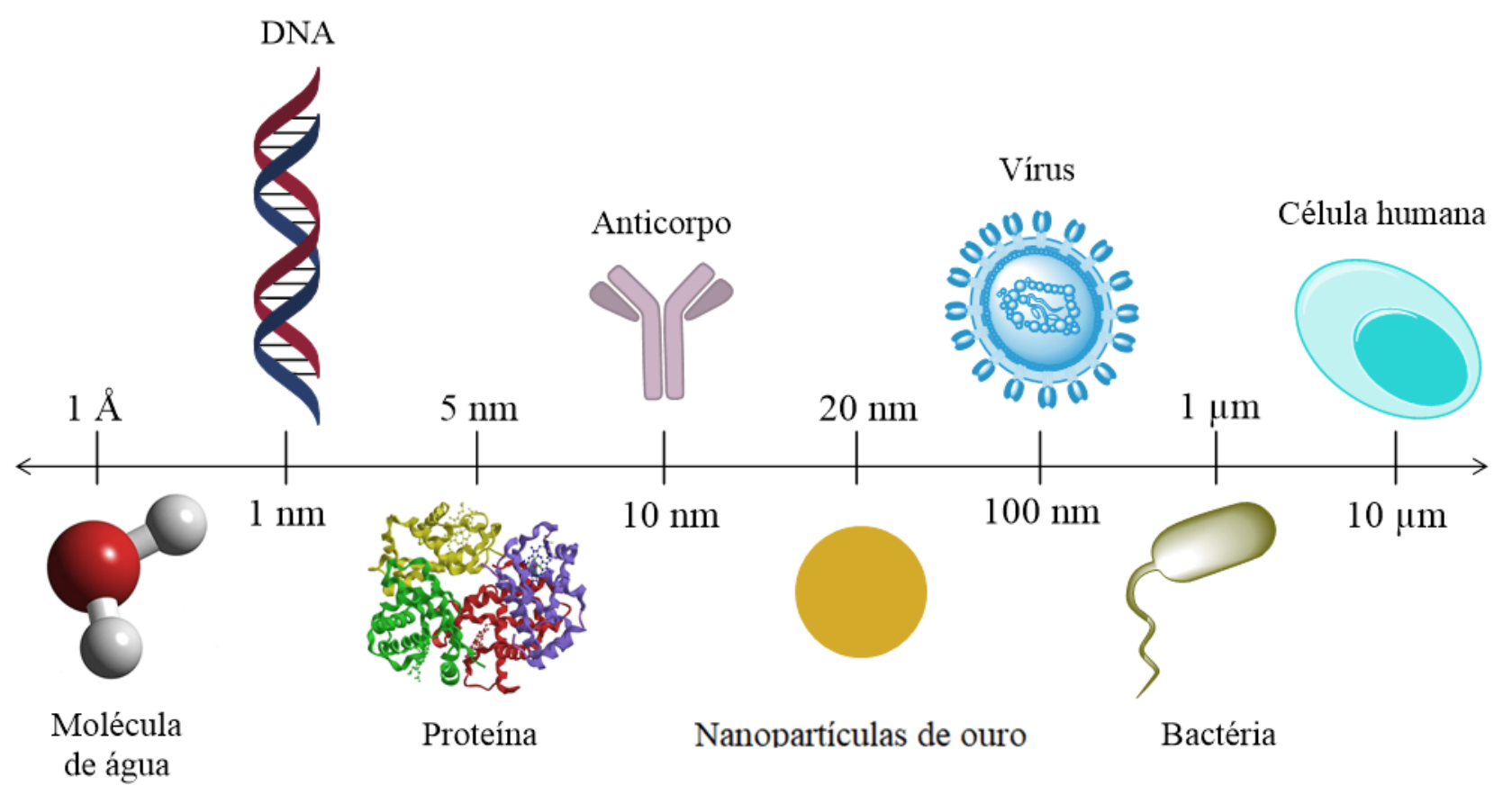

Figura 2. Tamanho de algumas estruturas relevantes na nanoescala.

Neste trabalho de revisão serão apresentadas algumas definições e conceitos fundamentais relacionados aos nanocarreadores, em especial suas características, métodos de preparação e design estrutural que possibilitam seu uso na entrega de fármacos para células-alvo, visando o tratamento de doenças como o câncer. Também serão apresentadas as características principais que os nanocarreadores, ou sistemas de liberação de fármacos, precisam apresentar para que possam ser efetivos, bem como os principais desafios e como estão sendo superados. Em outras palavras, serão apresentadas as principais estratégias que vêm sendo desenvolvidas e reportadas na literatura de modo a gerar sistemas eficientes de entrega de fármacos.

\section{Nanocarreadores: Definições e fundamentos}

Diferentes necessidades clínicas e enfermidades que acometem os seres humanos exigem diferentes tipos de sistemas de entrega de medicamentos. Nanomateriais têm sido amplamente estudados para desenvolver sistemas de entrega de fármacos inteligentes que 
atuem de maneira seletiva, liberando o fármaco nos locais desejados. ${ }^{5}$ Diversos estudos reportados nos últimos anos mostram que a nanotecnologia pode contribuir para o tratamento de doenças. E, de fato, já existem diversas formulações cosméticas e farmacêuticas com nanotecnologia embarcada disponíveis para uso clinico., ${ }^{4,7}$

\section{a) Tipos de nanocarreadores}

Os nanocarreadores podem ser classificados em três tipos principais, os baseados em a) nanopartículas metálicas e b) de óxidos metálicos, e c) nanopartículas geradas por automontagem de surfactantes e polímeros. Dentre eles o segundo pode ser subdivido em dois grupos: nanomateriais onde b1) as moléculas bioativas encontram-se ligadas a superfície, e b2) inseridas nos poros ou intercaladas nas regiões interlamelares. Por outro lado, as nanopartículas preparadas com surfactantes e polímeros podem ser classificadas, dependendo do tipo de estrutura básica formada, em c1) micelas e micro/nanoemulsões, c2) nanoesferas, nanopartículas lipídicas e nanocápsulas, e c3) lipossomas (Figura 3). ${ }^{8,9}$

As nanopartículas metálicas podem ser sintetizadas por rotas fotoquímicas, eletroquímicas, crescimento dirigido por modelo e processos de síntese verdes, que se destacam devido às suas propriedades favoráveis ao meio ambiente (Rout2018). Depois de obtidas, as nanopartículas metálicas podem ligar fármacos por adsorção, e por esta razão processos de adsorção e de dessorção são importantes para o mecanismo de drug delivery nesses nanossistemas. Neste sentido, é importante considerar a relação entre superfície/volume e as propriedades físico-químicas da superfície das nanopartículas metálicas, já que esses são os fatores que determinam sua capacidade de adsorção., ${ }^{2,6}$ Dentre as nanopartículas metálicas que têm sido utilizadas como nanocarreadores, as de ouro se destacam, e podem ser preparadas tanto a partir dos seus sais e compostos (abordagens bottom-up) bem como por processos de cominuição de ouro metálico (abordagem topdown). Seu sucesso se deve principalmente a estabilidade química do núcleo de ouro bem como da facilidade de ligação de diferentes biomoléculas tais como enzimas, carboidratos, 
peptídeos e proteínas na superfície das nanopartículas, possibilitando o transporte efetivo das mesmas para as células-alvo. ${ }^{9}$

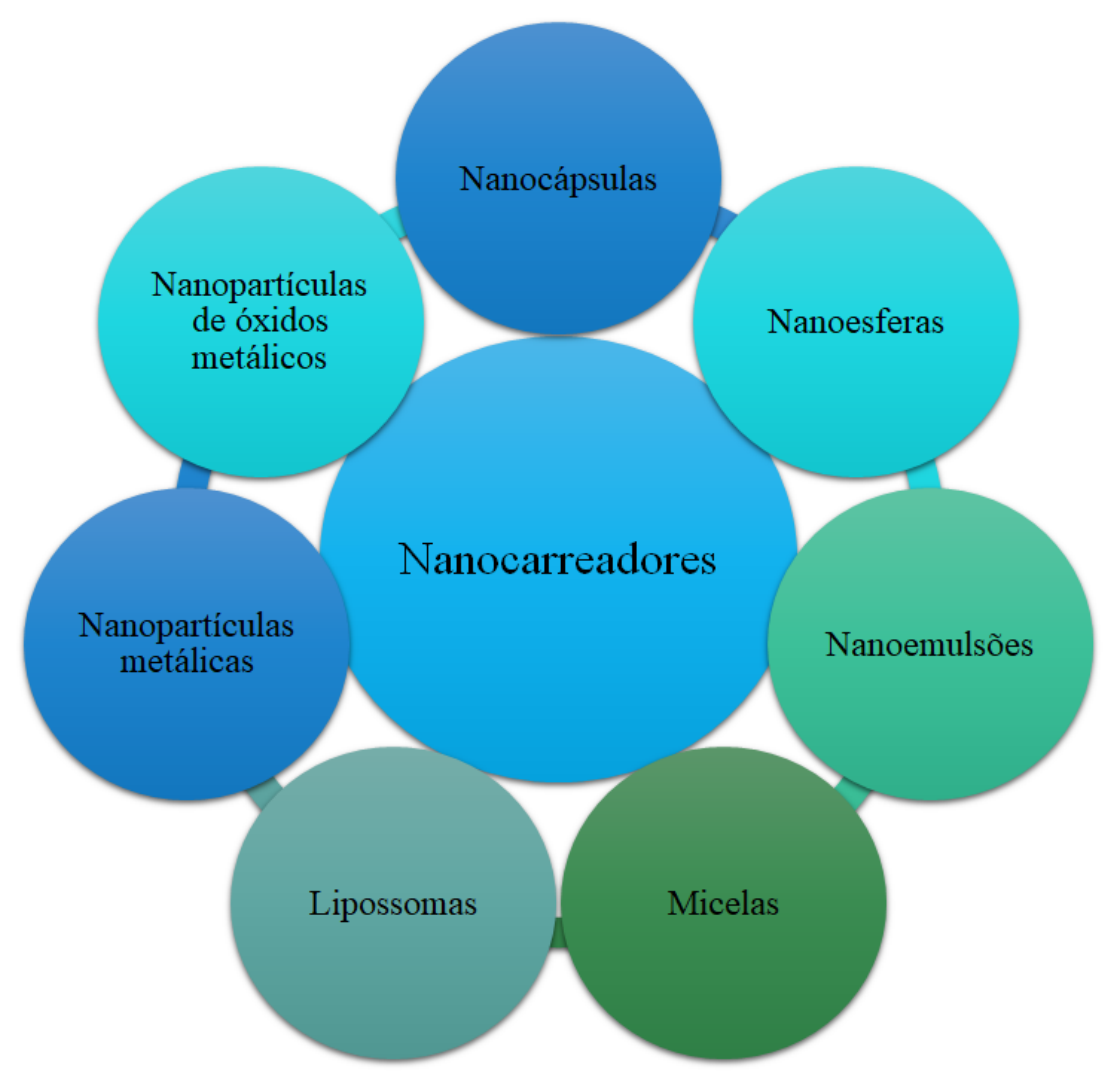

Figura 3: Esquema mostrando os diferentes tipos de nanomateriais que podem ser utilizados como nanocarreadores de fármacos.

Os mecanismos de adsorção e dessorção também são importantes para as nanopartículas de óxidos metálicos, já que elas também podem carregar fármacos em sua superfície por adsorção. Entre as nanopartículas de óxidos metálicos, as de óxido de ferro superparamagnéticas recobertas por polímeros merecem destaque devido ao seu pequeno tamanho (que permite maior internalização celular), propriedades magnéticas e biocompatibilidade. Tais propriedades possibilitam sua utilização em diversas aplicações farmacêuticas e/ou biomédicas. Essas nanopartículas podem, por exemplo, ser utilizadas 
como agentes de contraste para obtenção de imagens por ressonância magnética, na administração de fármacos de maneira direcional, tratamento por hipertermia, detecção precoce de inflamação, além de diagnóstico de doenças infecciosas como tuberculose, câncer, diabetes e aterosclerose. ${ }^{6,9,10}$ Um artigo de revisão da literatura publicado por Kawassaki et. al. (2021) aborda com detalhes as propriedades e as perspectivas atuais sobre nanopartículas de titânio e de óxido de ferro inteligentes para diagnóstico e tratamento de doenças. $^{3}$

As micelas são estruturas auto-montadas de moléculas anfifílicas (surfactantes moleculares e poliméricos) cujo processo de formação em fase aquosa são dirigidas principalmente por interações de van der Walls entre as cadeias de hidrocarbonetos, gerando domínios hidrofóbicos separados do meio hidrofílico. ${ }^{2}$ Nas micelas formadas em meio aquoso, a cauda apolar (hidrofóbica) está sempre voltada para o centro e cabeça polar (hidrofílica) fica em contato com o solvente polar. Além disso, em um solvente apolar é possível obter a micela inversa onde as cadeias apolares estão voltados para fora. As características da solução, como temperatura, força iônica e pH, e a natureza da molécula anfifílica determinam o tamanho e a forma das micelas produzidas quando a concentração de surfactante estiver acima da concentração micelar crítica, a concentração mínima necessária para a formação dessas estruturas. ${ }^{9,11}$

Por outro lado, as nanopartículas poliméricas podem ser classificadas como nanoesferas ou nanocápsulas dependendo das características da sua estrutura interna, sendo que as nanocápsulas possuem um núcleo sólido/líquido e as nanoesferas possuem uma estrutura regular que é composta por uma matrix polimérica sólida. ${ }^{12}$

Existem 3 métodos principais para obtenção de nanoesferas e nanocápsulas poliméricas: o método de deposição interfacial, o método "template" e o método camada sobre camada. Sendo que não existem inovações significativas nos últimos anos com relação à novas rotas de obtenção de nanocápsulas poliméricas. Todavia os métodos já conhecidos têm sido muito explorados para a preparação de nanomateriais utilizando-se diferentes materiais com 
intuito de satisfazer as diferentes características que os sistemas de entrega de drogas devem apresentar de acordo com o tipo de fármaco e a via de administração. ${ }^{12,13}$ Além disso, diversos tipos de polímeros naturais e sintéticos, que idealmente devem ser biocompatíveis e biodegradáveis, estão sendo utilizados no preparo de nanopartículas poliméricas. As principais vantagens desse nanocarreador comparado aos demais é sua versatilidade, já que eles podem utilizar diferentes tipos de polímero, dependendo do fármaco que se deseja encapsular, sua estabilidade em diferentes meios e a velocidade de liberação do fármaco em função do tempo. ${ }^{14}$ Uma descrição detalhada dos métodos de preparação de nanocápsulas poliméricas e os avanços conseguidos na última década é apresentada no artigo de revisão publicado por Deng e colaboradores. ${ }^{12}$

Por sua vez, os lipossomas são estruturas geralmente formadas por bicamadas fosfolipídicas, ou seja, moléculas anfifílicas que possuem um grupo fosfato hidrofílico e duas caudas de ácido graxo hidrofóbicas. Quando essas moléculas são colocadas em uma solução aquosa elas se auto-organizam formando uma bicamada lipídica de modo a minimizar a interação com a água (as caudas hidrofóbicas de duas monocamadas se juntam). O processo de formação é dirigido por força de van der Waals, ligações de hidrogênio e interações eletrostáticas. Lipossomas possuem estrutura semelhante à da membrana celular, sendo por esta razão mais estáveis que micelas formadas por surfactantes com uma única cauda hidrofóbica. ${ }^{2,11,15}$

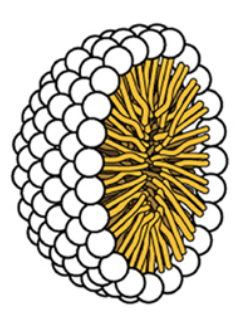

Micela

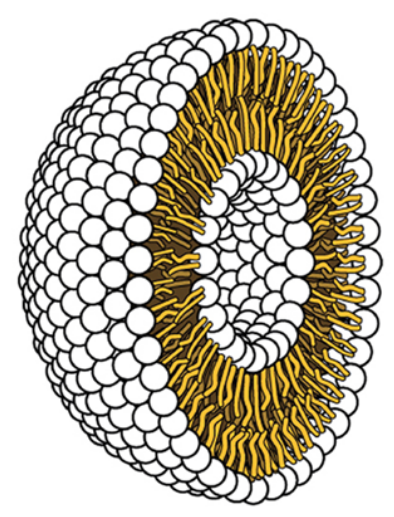

Lipossomo

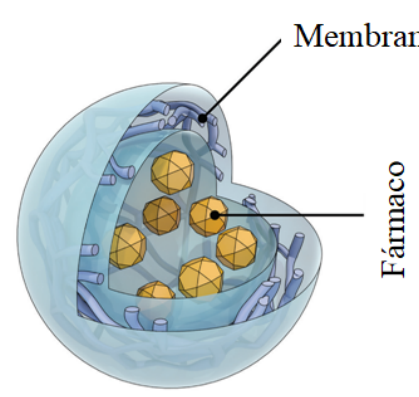

Nanocápsula

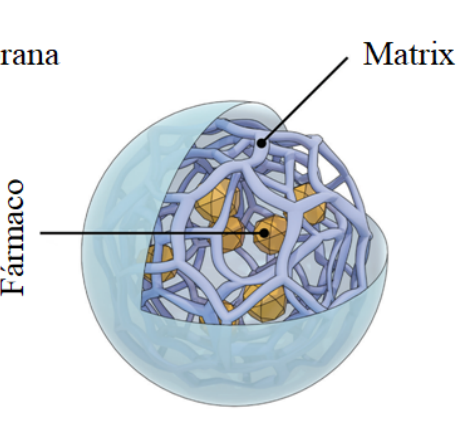

Nanoesfera 
Figura 3. Representação esquemática da estrutura de micelas, lipossomas, nanocápsulas e nanosferas. ${ }^{16,17}$

\section{b) Vetorização Molecular}

O controle da química de superfície dos nanomateriais é fundamental ao projetar nanobiomateriais funcionais inteligentes para aplicações médicas e biomédicas, já que a superfície desses materiais atua como a interface com o ambiente circundante, desempenhando um papel fundamental nas interações e determinando o destino biológico dos nanomateriais. ${ }^{3}$ Por esta razão os nanocarreadores podem ser funcionalizados para aumentar sua biocompatibilidade e incorporação de moléculas vetorizantes com capacidade de direcionar o nanocarreador para que seu conteúdo seja entregue e concentrado nos alvos biológicos desejados. ${ }^{9,18}$ A vetorização e a liberação das moléculas bioativas em sítios específicos (alvos) é um dos processos mais fundamentais para o tratamento de doenças pois maximizam a efetividade e minimizam os efeitos colaterais, assim viabilizando a terapia molecular personalizada. Além disso é importante salientar que as pequenas dimensões dos nanocarreadores são fundamentais para que eles possam alcançar os alvos desejados e realizar a entrega do princípio ativo de maneira precisa e homogênea, promovendo a funcionalidade e o desempenho terapêutico intensificado dos nanomateriais, enquanto se controla outras propriedades complementares relevantes. ${ }^{8,18}$ Alguns dos compostos que podem ser empregados para este objetivo são aptâmeros, peptídeos, anticorpos e antígenos. ${ }^{3}$

c) Vias de administração

Dentre as vias de administração, provavelmente, a administração intravenosa é a que oferece os maiores desafios, pois a formulação concentrada entra em contato com o sangue onde vai interagir com as diversas espécies/partículas nela presentes. Logo, podem sofrer modificações em suas propriedades, ou levar a formação de aglomerados capazes de induzir 
trombose. Assim, apesar de parecer uma tarefa relativamente simples, vários são os obstáculos a serem superados. De fato os nanocarreadores devem a) nanopartículas/nanocápsulas devem ser compatíveis com os fluidos corpóreos de modo a evitar aglomeração e mudanças importantes de propriedades em função do efeito corona, b) ser invisível ao sistema imune responsável pela proteção do nosso organismo, que quando ativado leva a eliminação de corpos estranhos por processos de fagocitose, endocitose e pinocitose, c) ter tempo suficiente de circulação evitando a eliminação pelos sistemas de excreção, principalmente a renal, d) ser vetorizados para as células/tecidos tumorais, e) ser incorporados pelas células/tecidos tumorais, e f) liberar os fármacos no meio intracelular e provocar a morte celular por apoptose ou necrose. ${ }^{3,15,18}$

\section{Nanocarreadores: Desenvolvimentos Recentes}

Muitos trabalhos focados na preparação de nanocarreadores mais eficientes e biocompatíveis vêm sendo publicados nos últimos anos, explorando diversas estratégias para melhorar/adequar as propriedades dos nanocarreadores. Por exemplo, em um trabalho desenvolvido por Romano et. al. (2020) foram obtidas uma série de nanopartículas de óxido de ferro superparamagnéticas (SPIONs) conjugadas com ibuprofeno (ibu) e fosfato de glicerol (glicerol) ou ibu e glicose-1-fosfato (Figura 3) com o objetivo de avaliar a atividade anti-inflamatória desses nanomateriais, ${ }^{19}$ constatada nos conjugados SPION-glicerol fosfato/ibu pela inibição da liberação de óxido nítrico (NO) induzida por lipopolissacarídeos em macrófagos, mesmo em baixas concentrações. 

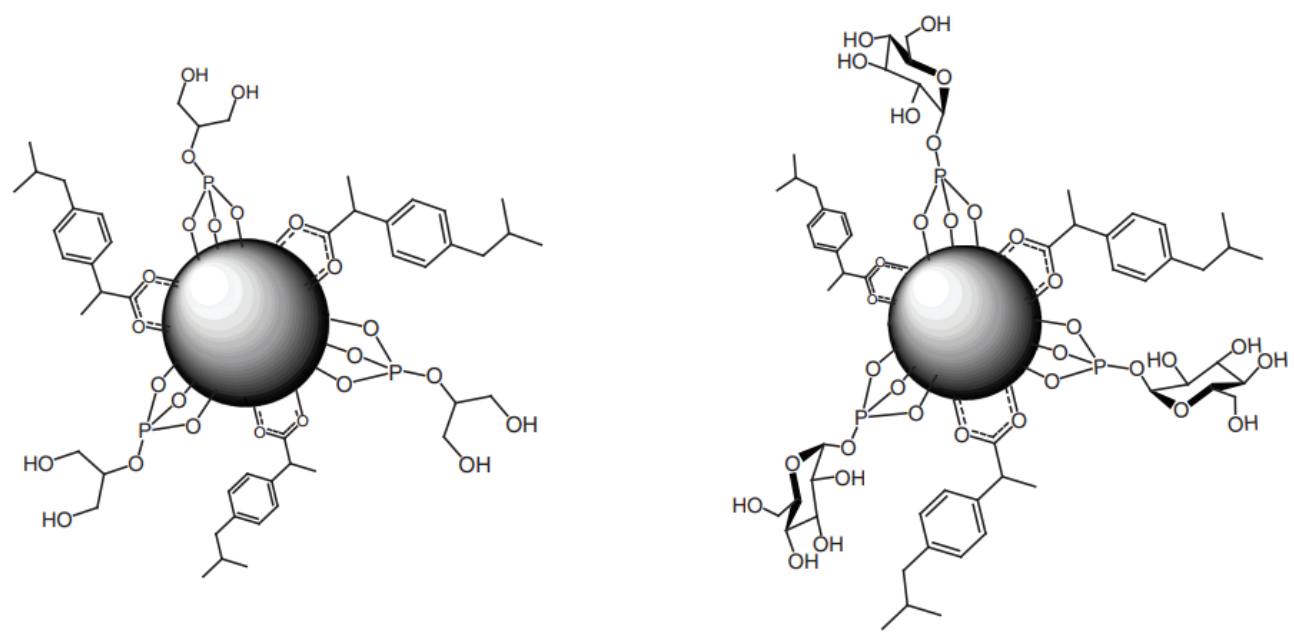

Figura 3: Nanopartículas de óxido de ferro superparamagnéticas conjugadas com ibuprofeno e glicerol fosfato (esquerda) e ibuprofeno e glicose-1-fosfato (direita). ${ }^{19}$

Além disso, há diversas formulações baseadas em nanocarreadores que foram aprovados pelas agências reguladoras de medicamentos, como a Food and Drug Administration (FDA) dos Estados Unidos e a European Medicines Agency (EMA) (Tabela 1), demonstrando o grande potencial de aplicação dos mesmos para o desenvolvimento de diversas aplicações e produtos, especialmente voltados para a área biomédica. ${ }^{3,6}$

Tabela 1. Exemplos de nanocarreadores comerciais, sua eficácia terapêutica e aprovação por agência reguladora. ${ }^{6}$

Nome (tipo) $\quad$ Eficácia terapêutica e diagnóstica $\quad$ Aprovação $\quad$ Referências

\begin{tabular}{|c|c|c|c|}
\hline $\begin{array}{l}\text { Feraheme (AMAG)/Rienso } \\
\text { (Takeda)/ Ferumoxytol (ferro }\end{array}$ & $\begin{array}{l}\text { Deficiência de ferro em pacientes } \\
\text { com doença crônica (doença renal) }\end{array}$ & FDA (2009) & 20 \\
\hline poliglucose & & & \\
\hline Carboximetiléter colóide) & & & \\
\hline Injectafter/Ferinject (Vifor) & Anemia ferropriva & FDA (2013) & 21 \\
\hline
\end{tabular}


(ferro carboximaltose colóide)

Diprivan (propofol

lipossomal)

AmBisome (Gilead Sciences) (anfotericina B lipossomal)
Indução e manutenção de sedação

FDA (1989)

21 ou anestesia

Meningite criptocócica em FDA (1997) 22 pacientes infectados com HIV Infecções por Aspergillus, Candida e/ou espécies de Cryptococcus (secundária) e parasita da leishmaniose visceral em pacientes imunocomprometidos

Visudyne (Bausch e Lomb) Tratamento de neovascularização (verteporfina lipossomal) coróide subfoveal de degeneração macular relacionada à idade, FDA (2000)

histoplasmose patológica ou ocular

\section{e EMA}

(2000)

Além disso diversos esforços vêm sendo realizados no desenvolvimento de novas formulações com o objetivo de nanoencapsular ativos para que eles possam ser entregues de maneira mais efetiva ao local desejado e, também, para que seja necessária uma menor dose do fármaco para se alcançar o efeito desejado, assim diminuindo possíveis efeitos colaterais. Por exemplo, Matteis et. al. (2018), realizaram o nanoencapsulamento da bedaquilina o primeiro medicamento eficaz contra a tuberculose desenvolvido nos últimos 40 anos. Porém, os efeitos colaterais da bedaquilina são tão perigosos que, no momento, este medicamento só é prescrito quando não existem outras opções de tratamento disponíveis. Por esta razão, novos métodos baseados em nanotecnologia podem ser particularmente relevantes para aumentar a concentração do fármaco somente no local da infecção, de modo a reduzir as doses necessárias e, consequentemente, os efeitos colaterais graves ampliando sua aplicação clínica. O medicamento foi encapsulado em dois tipos de nanocarreadores, 
nanopartículas lipídicas e nanocápsulas à base de quitosana, que apresentaram alta eficiência de encapsulação do fármaco. A eficácia das nanoformulações, bem como a excelente compatibilidade de ambos com células animais, foi demonstrada in vitro contra Mycobacterium tuberculosis. ${ }^{24}$

Há também estudos interessantes, como o desenvolvido por Sandri et. al. (2019), que demonstrou que nanocápsulas de núcleo lipídico (LNCs) de poli-( $\varepsilon$-caprolactona), além de funcionarem como transportadores de drogas eficientes e livres exibem efeitos terapêuticos, tem a capacidade de inibir o crescimento tumoral e a atividade de neutrófilos. Os LNCs penetram rapidamente nos leucócitos sem apresentar citotoxicidade, inibindo a proliferação de linfócitos induzida por mitógenos, a liberação de citocinas e a migração de leucócitos sob estimulação inflamatória, que foram associadas à inibição da via da MAP quinase e ao influxo de cálcio intracelular. Ou seja, os resultados indicaram que os LNCs podem agir como agentes reguladores negativos em células do sistema imunológico, sugerindo que as próprias nanopartículas vazias, ou carregadas com fármacos, podem interromper os processos inflamatórios. ${ }^{7}$

\section{CONCLUSÕES}

Nanocarreadores e nanossistemas de entrega de fármacos cada vez mais sofisticados vem sendo desenvolvidos de modo a atender as necessidades geradas pelo grande número de doenças que acometem os organismos vivos, em especial os seres humanos. Todavia, para que possam ser utilizados clinicamente devem apresentar propriedades tais como biocompatibilidade, tempo prolongado de circulação e capacidade de vetorização para alvos específicos, além de capacidade de entregar seu conteúdo gerando os efeitos desejados, serem fáceis de preparar, manipular e economicamente competitivos no mercado. Para isso, nanomateriais estão sendo desenvolvidos com diversos tipos de materiais tais como metais, óxidos metálicos, polímeros/biopolímeros e surfactantes de modo a melhorar a eficiência de moléculas bioativas, tornando-as dispersáveis em água e biocompatíveis, aumentando a 
estabilidade e a biodistribuição, bem como diminuindo a dose e os efeitos colaterais. Para isso é preciso gerar "nanopartículas fantasmas", nanomateriais inteligentes que não sejam reconhecidas pelo sistema imune e sejam mais lentamente filtradas pelo sistema de excreção, para que possam ser direcionadas para os alvos, onde irão promover a ação desejada. O potencial de aplicação de sistemas de entrega controlada e direcionada de fármacos é enorme, mas suas propriedades devem ser validadas não somente através de ensaios in vitro mas também por ensaios in vivo, a fim de verificar a toxicidade, eficácia e segurança dos potenciais agentes terapêuticos em modelos mais complexos e similares aos de aplicação real.

\section{REFERÊNCIAS}

1 S. Bayda, M. Adeel, T. Tuccinardi, M. Cordani and F. Rizzolio, Molecules, 2019, 25, 112.

2 N. Wang, X. Cheng, N. Li, H. Wang and H. Chen, Adv. Healthc. Mater., 2019, 8, 1801002.

3 R. K. Kawassaki, M. Romano, N. Dietrich and K. Araki, Front. Nanotechnol., 2021, 3, 735434.

4 D. K. Deda and K. Araki, J. Braz. Chem. Soc., , DOI:10.5935/0103-5053.20150316.

5 Z. Shi, Y. Zhou, T. Fan, Y. Lin, H. Zhang and L. Mei, Smart Mater. Med., 2020, 1, 32-47.

6 G. K. Rout, H.-S. Shin, S. Gouda, S. Sahoo, G. Das, L. F. Fraceto and J. K. Patra, Artif. Cells Nanomedicine Biotechnol., 2018, 46, 1053-1062.

7 S. Sandri, C. B. Hebeda, R. A. Loiola, S. Calgaroto, M. K. Uchiyama, K. Araki, L. A. Frank, K. Paese, S. S. Guterres, A. R. Pohlmann and S. H. P. Farsky, Nanomed., 2019, 14, 1429-1442.

8 S. Su and P. M. Kang, Pharmaceutics, 2020, 12, 837.

9 M. Chamundeeswari, J. Jeslin and M. L. Verma, Environ. Chem. Lett., 2019, 17, 849-865.

10 M. K. Uchiyama, C. B. Hebeda, S. Sandri, M. de Paula-Silva, M. Romano, R. M. Cardoso, S. H. Toma, K. Araki and S. H. Farsky, Nanomed., 2021, 16, 741-758.

11 R. Ahmad, S. Srivastava, S. Ghosh and S. K. Khare, Colloids Surf. B Biointerfaces, 2021, 197, 111389.

12 S. Deng, M. R. Gigliobianco, R. Censi and P. Di Martino, Nanomaterials, 2020, 10, 847.

13 A. Rehman, Q. Tong, S. M. Jafari, E. Assadpour, Q. Shehzad, R. M. Aadil, M. W. Iqbal, M. M.

A. Rashed, B. S. Mushtaq and W. Ashraf, Adv. Colloid Interface Sci., 2020, 275, 102048.

14D. K. Mishra, R. Shandilya and P. K. Mishra, Nanomedicine Nanotechnol. Biol. Med., 2018, 14, 2023-2050.

15 M. Elmowafy, Colloids Surf. B Biointerfaces, 2021, 203, 111748.

16D. Bitounis, R. Fanciullino, A. Iliadis and J. Ciccolini, ISRN Pharm., 2012, 2012, 1-11.

17 G. Suffredini, J. E. East and L. M. Levy, Am. J. Neuroradiol., 2014, 35, 1246-1253.

18 A. Shah, S. Aftab, J. Nisar, M. N. Ashiq and F. J. Iftikhar, J. Drug Deliv. Sci. Technol., 2021, 62, 102426. 
19M. Romano, M. K. Uchiyama, R. M. Cardoso, S. H. Toma, M. S. Baptista and K. Araki, Nanomed., 2020, 15, 2475-2492.

20 M. Auerbach and J. W. Adamson, Am. J. Hematol., 2016, 91, 31-38.

21 M. G. Braz, L. G. Braz, C. M. M. Freire, L. M. C. Lucio, J. R. C. Braz, G. Tang, D. M. F.

Salvadori and K.-J. Yeum, Medicine (Baltimore), 2015, 94, e1266.

22 A. C. Anselmo and S. Mitragotri, Bioeng. Transl. Med., 2016, 1, 10-29.

23 Y. Min, J. M. Caster, M. J. Eblan and A. Z. Wang, Chem. Rev., 2015, 115, 11147-11190.

24L. De Matteis, D. Jary, A. Lucía, S. García-Embid, I. Serrano-Sevilla, D. Pérez, J. A. Ainsa, F. P.

Navarro and J. M. de la Fuente, Chem. Eng. J., 2018, 340, 181-191. 